\title{
A Case of a Giant Ovarian Mass in a Teenager: Case Report and Review of Literature
}

\author{
Robert Hurwitz ${ }^{*}$ \\ Department of Radiology, University of Nevada, Las Vegas, USA
}

"Corresponding Author: Dr. Robert Hurwitz, Department of Radiology, University of Nevada, Las Vegas 89123, USA, Tel: +19494221453; E-mail: Bob95Fox@aol.com

Received: 22 October 2018; Accepted: 01 November 2018; Published: 16 November 2018

\begin{abstract}
Ovarian tumors in children are uncommon, whether benign or malignant. The diagnosis must be considered, however, when there is unexplained pelvic pain. A palpable mass further raises suspicion and warrants prompt pelvic ultrasound. On occasion, precocious puberty and hirsutism will assist in the clinical evaluation. Surgery is the primary treatment for all ovarian tumors. Attempt at ovarian salvage to preserve fertility is essential. A case report follows, describing a large benign right ovarian tumor whose diagnosis was delayed because of unrelated clinical problems.
\end{abstract}

Keywords: Ovarian Tumors; Abdominal pain; Computed tomography; Pediatric tumors

\section{Introduction}

Ovarian tumors are uncommon in childhood and the teenage years [1, 2]. They can be either benign or malignant. Most present with pain. With further tumor growth, however, a mass becomes palpable. Ultrasound is the preferred modality for detection. Computed tomography (CT) and more recently, magnetic resonance imaging (MRI) both play an important role in detection and staging [3]. A case report follows which describes a $14 \mathrm{~cm}$. fibrothecoma in a 15 year old teenage girl. This was likely present seven years prior but became clinically obvious following the onset of puberty.

\section{Case Report}

An eight-year-old female presented with abdominal pain and fever. Ultrasound revealed a ruptured appendix. Emergency appendectomy was successful. Persistent pain in subsequent months prompted computed tomography of 
the abdomen and pelvis using low dose, non-contrast technique. This revealed a $2.0 \mathrm{~cm}$ uncomplicated fluid collection adjacent to the cecum. Careful clinical follow-up showed no leukocytosis or fever. The CT findings were therefore presumed to represent a benign post-op seroma (Figure 1).

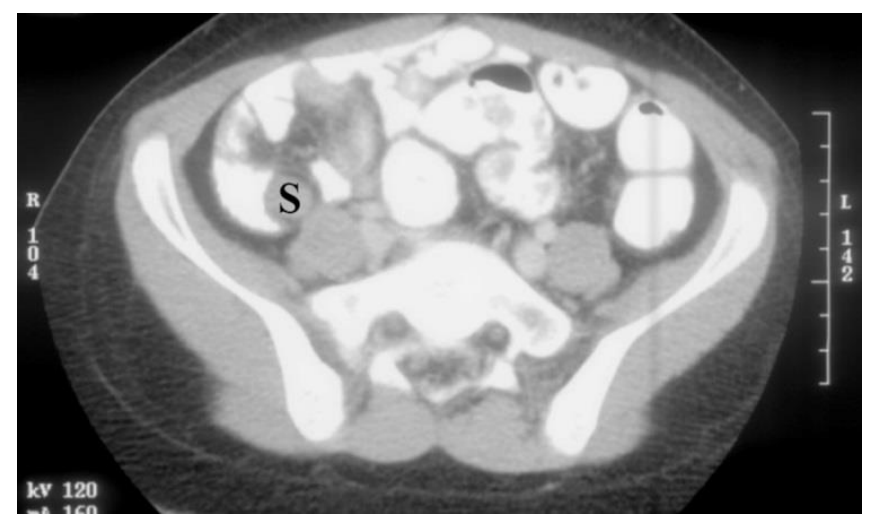

Figure 1: CT of pelvis after appendectomy at age 8. ( $\mathrm{S}$ indicates a benign post-operative seroma).

Only in retrospect could one also identify a small solid mass arising from the superior aspect of the nearby right ovary. Seven years followed, with numerous childhood illnesses of recurrent otitis and pharyngitis. On each occasion, the patient complained of intermittent abdominal pain. On physical examination there was no rebound tenderness and no palpable mass. No signs of precocious puberty were observed. At 15 years of age, the pain became intense. CT scan revealed a $14.0 \mathrm{~cm}$ solid mass in the midline pelvis, likely arising from the right ovary (Figure 2). There was mild bilateral hydronephrosis and mild ascites. Pelvic ultrasound confirmed this finding (Figure 3). Emergency surgery was performed. A $14.0 \mathrm{~cm}$ benign fibrothecoma was removed (Figure 4). There was torsion of the tumor mass. Both the right ovary and right fallopian tube required resection. The uterus and left ovary were both proven to be entirely normal.

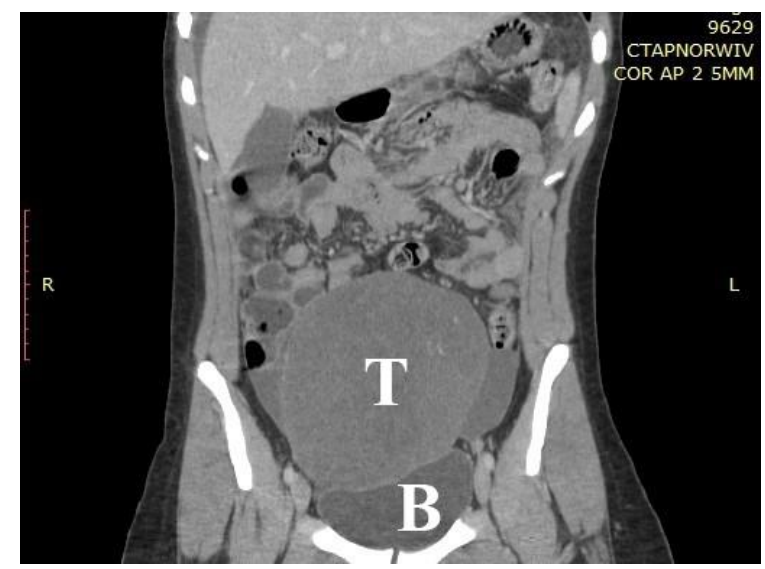

Figure 2: CT Scan at age 15. T indicates a large solid ovarian tumor; B labels the compressed urinary bladder. 


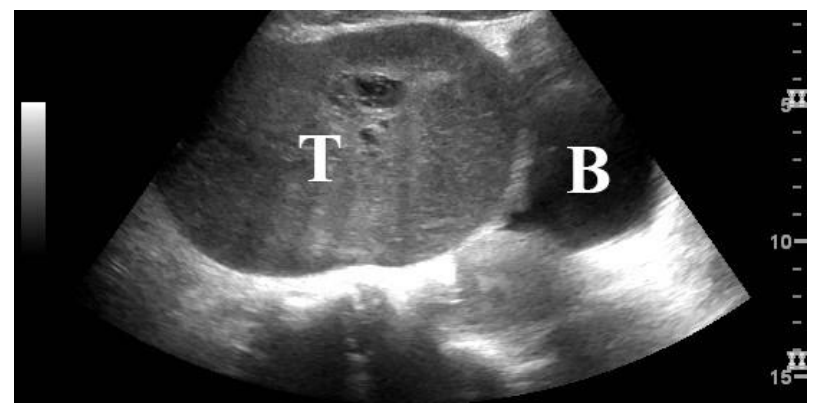

Figure 3: Sagittal ultrasound at age 15. T indicates a large solid ovarian tumor; B labels the compressed urinary bladder.

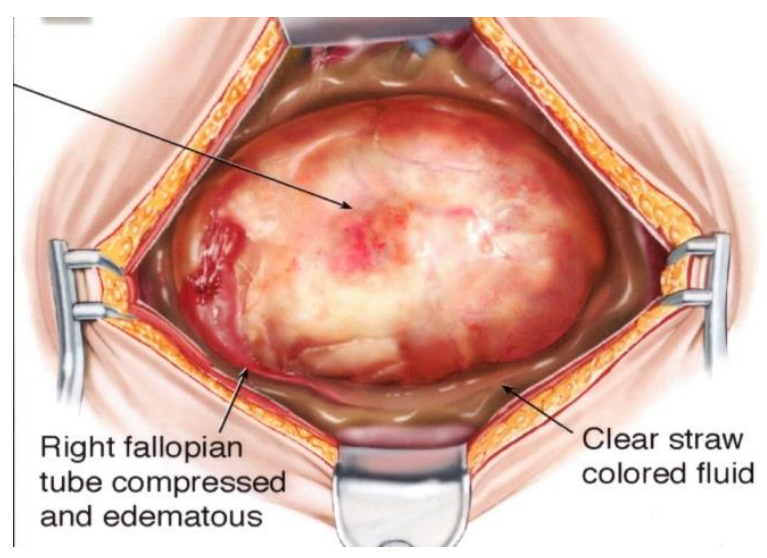

Figure 4: Drawing of surgical findings. Enormous benign ovarian fibrothecoma (indicated by the long arrow).

\section{Discussion}

Ovarian neoplasms in the pediatric and adolescent population represent 2.6 cases per 100,000 girls a year [1]. Despite being uncommon, tumors of the ovary in children are the most common gynecologic malignancy in this age group. Ovarian tumors in childhood include germ cell tumors, (GCTs), stromal tumors, sex cord-stromal tumors (SCSTs) and rare miscellaneous tumors [2]. Germ cell tumors are the most frequent, comprising 60-80\% of the frequency of lesions found [3,4]. Abdominal pain is the most common presenting symptom of ovarian tumors, whether benign or malignant. Pelvic tenderness may be due to torsion, hemorrhage or rupture of the tumor. Endocrine abnormalities can often be the first manifestation of hormone-producing tumors. Premenarchal precocious puberty or virilization should immediately raise the possibility of ovarian tumor.

Ultrasound is the best screening test for the pediatric group [3, 5]. Computed tomography, as in the case above, proved essential in an emergent presentation. Magnetic resonance imaging (MRI) is now a useful modality in children and avoids exposure to the radiation of CT [6]. This examination takes a longer time and may require sedation in a young child. Serum assays of tumor markers helps establish a differential diagnosis [4]. Such tests include $\beta$-hCG, AFP, LDH and CA-125 levels. Careful surgical planning is necessary to preserve fertility in girls 
and young women. Laparoscopic removal is the optimal approach. The fibrothecoma found in the Case Report above is normally found in adult women older than 30 years of age. The finding of ascites in this patient is a typical associated sign $[3,4]$.

While primary ovarian tumors in children and adolescents are uncommon, pelvic pain should always raise attention to this possibility. Ultrasound, CT and MRI are all useful imaging techniques to differentiate benign from malignant lesions. Selecting the appropriate surgical procedure is essential to preserve future fertility.

\section{Acknowledgment}

None

\section{Conflict of Interest}

The author declares that he has no conflict of interest

\section{References}

1. Breen JL, Maxson WS: Ovarian tumors in children and adolescents. Clin Obst and Gyn 20 (1977): 607623.

2. Enhren MD, Mhour GH, Isaacs H. Benign and malignant ovarian tumors in children and adolescents. Am J Surg 147 (1984): 339-344.

3. Athey PA, Malone RS. Sonography of Ovarian Fibromas/thecomas. J of Ultrasound Med 6 (1987): 431436.

4. Heo SK, Kim JW, Shin SS, et al. Review of ovarian tumors in children and adolescents. Radiographics 34 (2014): 1-42.

5. Bazot M, Ghossain MA, Buy JN, et al. Fibrothecomas of the ovary. J. Comp Assist Tomogr 17 (1993): 754-759.

6. Shinagare AB, Meylaerts LJ, Laury AR, et al. MRI features of ovarian fibroma and fibrothecoma. Am J of Roent 198 (2012): W296-W303.

Citation: Robert Hurwitz. A Case of a Giant Ovarian Mass in a Teenager: Case Report and Review of Literature. Archives of Clinical and Medical Case Reports 2 (2018): 227-230. 